\title{
Scientonomy 4
}

Palider, K. (2019). Scientonomy 3, 15-31

https://www.scientowiki.com/Palider (2019)

https://doi.org/10.33137/js.v3i0.33557

\section{Reasons in the Scientonomic Ontology}

\author{
Kye Palider
}

IHPST, University of Toronto

kye.palider@mail.utoronto.ca

\section{Keywords}

theoretical scientonomy, ontology of reasons, inference, implication, sufficient reason, support, reason, normative inference, sufficient reason theorem

\section{Abstract}

The question of how we come to accept new theories is a central area of inquiry in scientonomic discourse. However, there has yet to be a formal discussion of the subjective reasons an agent may have for accepting theories. This paper explores these epistemic reasons and constructs a historically sensitive definition of reason. This formulation takes an abstractionist stance towards the ontology of reasons and makes use of a composite basing relation. The descriptive and normative components of reasons are fully formulated in scientonomic terms through the application of the newly introduced notion of implication, and its separation from the notion of inference. In addition, the paper provides scientonomic definitions for sufficient reason, support, and normative inference. The fruitfulness of this formulation of reasons is illustrated by a few examples. 


\section{Introduction}

What does it mean for something to be a reason for accepting a theory? Epistemologists and philosophers have postulated countless competing answers to these questions. Reasons are also ubiquitous in science and the history of science. Furthermore, they are indispensable when speaking about why epistemic agents change their beliefs. Despite this, scientonomy has yet to offer an ontological account of reasons or their role in the dynamics of scientific change. The aim of this paper is to introduce reasons into the scientonomic ontology in a way that will be useful for historical purposes, relevant to epistemological literature on reasons, and ultimately serve as a fruitful component of a theory of scientific change.

The primary intended use of the account of reasons presented in this paper is understanding the historical reasons of epistemic agents for taking an epistemic stance towards an epistemic element. To be applicable to historical episodes, an account of reasons should not be anachronistic or objective in the sense of normative epistemology. It should be able to capture the subjective reasons of different epistemic agents in different time periods and be able to represent their reasons in a historically accurate manner. For instance, the reasons for not accepting heliocentrism from Galileo's observations should be historically sensitive rather than labelled as irrational or as bad reasoning.

The scientonomic ontology of reasons should also reflect the current philosophical literature on the topic. Thus, the ontology presented here is inspired by available epistemological accounts of reasons. Specifically, I will discuss a number of philosophical views on reasons and will modify and adapt them into the scientonomic context so as to maintain some basis of comparison and invite critique from epistemologists. In addition, I relate reasons to the notions of inference and implication.

Although this paper does not tackle the dynamics of reasons and does not introduce any new laws of scientific change, the ontology and definition of reasons presented in this paper will hopefully prove useful as a first step towards incorporating reasons into scientonomy.

This paper is organized as follows. I first discuss some of the central conceptions of reasons in the philosophical literature with the goal of distilling a notion of reasons most appropriate for scientonomy. I then express those views in already available scientonomic terms. I then present the definition of reason and discuss its consequences. Lastly, I illustrate this concept of reason with a few examples.

\section{Ontology of Reasons}

The philosophical literature here discussed will primarily concern epistemic reasons, i.e. reasons for holding beliefs. Explanatory reasons (or causes as they are sometimes called) for certain events transpiring will not be considered. Before exploring more robust definitions of epistemic reasons, I take the following preliminary definition (or intuition) of an epistemic reason. An epistemic reason is a motivation, and not simply explanation, for an agent to hold a belief or other doxastic attitude (Sylvan 2016b, p. 377).

So, what are reasons ontologically? Are reasons propositions, beliefs, or something else? There are three common views on the nature of reasons (Turri, 2009, p. 492):

- Factualism: Reasons are non-mental facts or states of affairs.

- Statism: Reasons are mental states.

- Abstractionism: Reasons are the propositional contents of the subject's mental states.

Factualism, although integrating well with logic, is certainly inappropriate in a historical context. Factualism purports that reasons can only be true statements. This allows one to work with reasons in an analytic fashion. As a factualist, one can check for validity, consistency, and other properties of interest when looking at reasons. However, the factualist must deny reasons that are not true, even if the agent believes them. Historical scientific theories (which were frequently used as reasons and reasoned for) are now simply considered false. However, a historian may want to talk of the reasons for certain historical agents' beliefs even if they included false scientific theories, but the factualist view would not permit calling non-factual states of affairs reasons. Appeals to the truth 
I work with an intuitive sense of epistemic. Reasons are to do with an agent's thinking, rather than truth, states of affairs, facts about the world, etc. They may be related to these states of affairs, but only through the agent's thinking. are also utopian and a historian cannot "check" if a reason is a fact as it is not truth-sensitive. Reasons are not alethic, they are epistemic. It is untenable for a formulation of reasons that is relevant in the history of science to be a

factualist formulation.

Statism, although compelling, reduces reasons to psychological or cognitive studies, whereas the epistemic reasons postulated herein are ones describing rationality. Bonjour (2003) makes it clear that it is impossible to take anything as a reason without at least being cognitively aware of the reason. It seems non-controversial to say

Rationality here is meant to be an intellectual phenomenon that does not have a readily available construction through psychology or neuroscience. It refers to aspects of thought rather than just mental states. to have a belief that she did receive presents from Santa Claus. If not, how could the non-mental proposition of her receiving presents be a reason? Hence, it seems that one cannot have a reason that is not a mental state. Furthermore, it seems that anything an agent has the ability to think about is fully determined by their mental/cognitive state. This seemingly leaves no room for any logic or propositional content to enter the picture. However, even though all reasons boil down to mental states, we can still study them from an epistemic viewpoint. Here, epistemic would refer to a higher-level study of reasoning than neuroscience, and perhaps an idealized study of reasoning that may be normative in and of itself, i.e. how we ought to reason. This epistemic form of reason is what is of particular interest to intellectual history. Ignoring propositional content makes the study of the reasoning of historical agents practically impossible, as we do not have neuroscientific models for reasoning, nor do historians have access to agents' mental states. As such, statism seems unfavourable as a historically pragmatic formulation of reason, even though it seems to be grounded in a modern physicalist viewpoint in neuroscience.

Abstractionism is the best of both worlds: it acknowledges that reasons are mental states, while allowing for analytic work to be done with reasons. Like factualism, abstractionism allow for direct logical work to be done relating the propositions of reasons and beliefs. This work may include checking for consistency of believed propositions, showing how a reason might entail a consequence or belief, and more. One advantage of abstractionism over factualism is that the abstractionist can consider false beliefs as reasons, while the factualist cannot. The abstractionist also recognizes that reasons are mental states held by agents, while considering it fruitful to study reasons in a logical or epistemological fashion that goes beyond a scientific study of mental states themselves. I will attempt to show the advantages of an abstractionist stance through an example in recent mathematics.

In 1994, Zhang gave a negative answer to the Busemann-Petty problem in four dimensions (Zhang, 1994). In his paper, Zhang proved lemmas and theorems, eventually reaching a proof of a negative answer. It would be fair to say that, as a result of this proof, Zhang believed that the Busemann-Petty problem had a negative answer. The factualist might say the reason for Zhang believing this is that he made a valid deduction of it. The statist, on the other hand, would have to identify the reason as some form of mental state pertaining to the proof given in Zhang's paper. The statist seems to have little to say about the reason for Zhang's belief, as they cannot access Zhang's mental state, while the factualist has a precise reason. However, later in 1998, Koldobsky published a paper reaching a positive result for the Busemann-Petty problem in four dimensions (Koldobsky, 1998). The factualist runs into a problem here. Either Zhang or Koldobsky's proof is incorrect. Zhang later found an error in his proof and acknowledged the positive result in 1999. At this point, the factualist may say that Zhang did not have a reason to believe the negative answer in 1994 but did have a reason to believe the positive answer in 1999. Yet, this is problematic: a historian would wish to speak of the original proof in 1994 as being a reason for Zhang's (incorrect) beliefs, but this is not possible for the factualist. The statist has no problem with the turn of events, as prior mental states are still the same, but the statist still does not have a comprehensive account of Zhang's reasons. Neither the factualist nor the statist can properly handle this scenario. 
The abstractionist, on the other hand, does not struggle with this case. For the abstractionist, the reason for Zhang believing a negative answer in 1994 was Zhang's belief in the validity of his proof. Although the proof was faulty, it was still a reason, and its propositional content is still relevant. The abstractionist can further analyze the situation by saying that a revision of the propositional content of Zhang's proof in 1999 caused him to adopt a different view, namely a positive answer to the Busemann-Petty problem. Since in 1999 Zhang no longer believed his original proof to be valid, he no longer had a reason to believe in a negative answer. The abstractionist offers a much more comprehensive analysis of historical reasons than the factualist or the statist. The factualist fails because they adopt an untenable alethic notion of reason, while the statist fails because they adopt an untenable physicalist notion of reason. The abstractionist is instead respectful of Zhang's doxastic states towards his proof(s) and may still talk of the propositional content of those doxastic states, just like the factualist. It seems that, for a historically pragmatic theory of reason, the epistemic ontology of abstractionism is the most suitable.

The abstractionist view can easily be formulated in scientonomic terms. Relevant mental states are doxastic attitudes which in scientonomic terms can be regarded as epistemic stances, while propositional content can be regarded as the theories towards which an epistemic stance is taken. So, in scientonomic terms, the abstractionist viewpoint can be stated as follows: ontologically, reasons are theories towards which an agent takes an epistemic stance. Obviously, not all theories are reasons, and we shall come to classify what makes a theory a reason.

\section{Basing Relations}

While it is clear that, ontologically, reasons are theories, we have yet to look at what makes a certain theory a reason. Canonically, a proposition $B$ is a reason for proposition $A$ if and only if $B$ satisfies a basing relation with $A$, i.e. $B$ is a basis of belief for $A$. The basing relation takes many forms, but it is commonly represented by statements of the form " $B$ supports $A$ " or "from $B$ one can infer $A$ " (Korcz, 1997, p. 171). I will discuss various views on the basing relation and then begin to form a scientonomic notion of basing relations.

First, we must acknowledge that inferences and reasons are intimately related. If $B$ is a reason for $A, B$ must in some shape or form infer $A$. There are generally two types of inference cited in the literature on basing relation: causal and doxastic. Under a causal theory of reasons, the reason $B$ literally causes the belief in $A$ (Korcz, 1997, pp. 172-175). A causal theory is not usually considered psychological or neurological in nature, but is instead based on epistemic terms, such as awareness of logical relations (Korcz, 2000, p. 534). In contrast with a causal theory of reasons, a doxastic theory of reasons involves an inference from a normative meta-belief that says $B$ is a good reason for believing $A$ (Korcz, 1997, pp. 180-187). Some theories of reasons involve both causal and doxastic components (Korcz, 2000; Gregory, 2016; Cunningham, 2019; Boghossian, 2018). In both cases, inferences play a crucial role in establishing what a reason is. However, I believe the causal/doxastic distinction is unclear and requires some discussion.

The distinction between causal and doxastic theories of reasons in $\operatorname{Korcz}(1997,2000)$ is a distinction between descriptive inferences and normative reasons. The causal inferences of causal theories are necessarily descriptive in nature and not normative at all. They suppose that certain beliefs (reasons) physically cause other beliefs rather than merely justifying them. The meta-beliefs of doxastic theories which deem certain reasons as good reasons are necessarily normative. They must say, at minimum, what a good reason should be. The meta-belief is then a normative criterion which must be satisfied in order for a belief to qualify as a good reason for some other belief. From this, we can see that causal theories of reasons are descriptive and doxastic theories of reasons are normative. Thus, the causal/doxastic distinction can be rebranded as a descriptive/normative distinction. This distinction allows for a cleaner analysis of composite views - views involving both causal and doxastic components.

The composite view in general can be presented as follows (Cunningham 2019, p. 3893; see also Lord \& Sylvan, 2019):

$S$ believes that $p$ for the good reason that $q$ iff (i) $S$ believes that $p$ because $S$ believes that $q$ (ii) $q$ is a normative reason for $S$ to believe that $p$. 
Note that a theory of what makes a reason "good" refers to what makes a reason for believing something rational. I do not offer a normative theory of reasons here, so I do not provide objective criteria for a reason being good, similar to those provided by Cunningham, Lord, and Sylvan.

Here (i) encapsulates the causal, i.e. descriptive, condition for a reason, and (ii) encapsulates the doxastic, i.e. normative, condition. The descriptive condition can be understood as some connection between beliefs, be it logical or cognitive. The normative condition can be understood as some normative statements (meta-beliefs) that justify the reason being a good basis for belief (Gregory, 2016, pp. 2292-2295). In his analysis of inferences, Boghossian (2018) presents an instance of the composite view with a similar distinction between, to use his language, basing and quality (Boghossian, 2018, p. 59):

B. (Basing) When you infer from $P$ to $C$, you establish the premises $P$ as your reason for believing the conclusion $C$, you establish them as the basis on which you believe $B$.

Q. (Quality) Given the basing fact, your belief can be assessed as resting on good or bad reasons.

It is also clear to Boghossian here that the basing is a fact (i.e. descriptive) while the quality typically involves some goals and is thus normative in nature. Boghossian expands the basing fact and inference into six components. He says that the thinker (Boghossian, 2018, p. 57):

a. Explicitly judges the premises of the inference;

b. Explicitly wonders, in the context of some particular inquiry, what else she has reason to believe, what other proposition the premises support.

c. Explicitly takes the premises to support the conclusion.

d. Knows the properties of the premises in virtue of which they support the conclusion (knows the epistemic principle that validates moving from the premises to the conclusion).

e. Believes the conclusion because she believes the premises.

f. Believes the conclusion because she takes the conclusion to be supported by the premises.

Here Boghossian spells out robust conditions for when an agent makes an inference. Certain points seem to be causal or descriptive, while others are doxastic or normative. However, the distinction is not so clear (e.g. "knowing in virtue of" the premises could be interpreted as normative rather than descriptive). In this way, he provides a theory of inference which remains rather vague. These actions of "judgment", "wondering", "support", "knowing in virtue of", and "believing because of believing the premises" are all rather difficult to define or even categorize. Clearly, these terms all mean different things for different agents. What Boghossian's account lacks is a precise language of epistemic entities and relations and how they vary from agent to agent. This is not a problem just for Boghossian's account, but for many others trying to formulate a basing relation. The current scientonomic ontology of epistemic entities and relations is one such framework in which a basing relation can be built. I shall begin to do this by providing a discussion of implication, followed by a discussion of inference, culminating in a presentation of a scientonomic basing relation.

\section{Implication}

Implication refers to the logical procedure of "reasoning" from one proposition to another (for a similar stance see Harman, 1986 and his account of deduction). The term reasoning is not precise enough, as it may be

Note that the word implication is used here for the lack of a better term. It has many different uses in the literature which may differ from that of this paper. I am open to replacing it with a better option, if one is suggested. interpreted as referring to a mental activity, whereas the term implication refers exclusively to a purely computational activity. Implication is merely a computational movement from one proposition to the next that does not require any agent. There are typically two forms of implication: deductive and inductive. This distinction will not be directly relevant for this paper, and I will use the term implication to refer to either deductions or inductions of any sort, so implication will not be restricted to purely deductive or inductive forms 
of reasoning. I will write that a proposition $P$ implies a proposition $Q$ with an arrow. A subscript may be adopted if the nature of the implication is ambiguous in the context.

$$
P \rightarrow{ }_{\alpha} Q
$$

Implications, as I will refer to them, are not necessarily related to the material implication/conditional; they are abstract and relative to the formalism/logic given to them. They are a type of relation between propositions and are a logical or proof-theoretic device. To say that $P$ implies $Q$ is equivalent to saying that $Q$ follows from $P$, i.e. there is an argument from $P$ to $Q$, or, again equivalently, that one can prove $Q$ from $P$. I will give the general definition of implication as a logical transition from one theory to another. Here, logical simply refers to something "rule-governed", with no explicit definition of

I make no commitment to any definition of logic. However, I do view logic as a rule-governed normative science. Perhaps the properties of non-triviality, argument formation, rules of implication, etc. are taken for granted in a logic, but no explicit stance is taken towards what is classified as a logic in this case. Furthermore, the use of the term proposition may also be inappropriate in certain contexts, but it can be loosely interpreted as a "concept" or a linguistic entity (sentence). The scientonomic view of theories as sets of propositions is implicit here. These topics, although begging for elaboration, are not the focus of this paper directly. what this encompasses (it will be primitive in this paper), and transition can be interpreted as a primitive connection between two propositions, a type of computation or connection.

Implication $\equiv$

A logical transition from one theory to another.

Although logic may be viewed as normative, implication is a purely descriptive relation. It is now commonplace in philosophy to view logic as normative, i.e. telling us how we ought to reason (Steinberger, 2017). This applies both to inductive and deductive logic, making them both normative (Huber, 2017, pp. 508, 520). One would then think that implication is also normative, but this is not the case. There is a difference between the mere computational procedure and the process of belief revision. Harman (1986) viewed this as a distinction between implication and inference. Implications are tools for generating new conclusions, while inferences (not in the sense of a logical rule of inference, but in the sense of an epistemic agent making an inference) are forms of belief revision, i.e. changes in knowledge. An agent will

In addition, one can distinguish between the purely alethic notion of logic and the epistemic notion of logic (MacFarlane, 2004, pp. 1-2). The alethic notion is concerned with truthpreservation, deductive closures, and generating new conclusions, while the epistemic notion is the normative component that drives the agent's change in knowledge. Here, implication would simply refer to the alethic notion of logic. theories, but implications alone, as will soon be seen, not lead to acceptance or rejection of theories. Thus, it must be acknowledged that implication is a purely logical

(computational) procedure that tends to generate more theories, while inference is an epistemic activity that may lead to theory acceptance or rejection. Therefore, in scientonomic terms, the statement of implication is descriptive.

It is very tempting to conflate implication with truth-preservation or logical necessity, but implication may have nothing to do with either. In the classical sense, if a proposition $A$ implies a proposition $B$, then if $A$ is true, so is $B$, i.e. the notion of implication of classical logic is truth-preserving, as the truth of $A$ logically necessitates the truth of $B$. The notion of implication discussed hereafter is not as strict. It is merely a transition from one theory to another via some accepted rules of implication. These rules of implications need not involve any form of logical necessity or truth-preservation. They can be much weaker, as they usually are in inductive implications. For example, one could use some inductive logic to imply that all swans are white from a finite observation of white swans, even if this is not a truth-preserving implication. This is not to say that the agent believes this form of inductive logic to be truth-preserving and is mistaken about that. All that is being said is that the agent, under some rule of implication, manages to transition from the theory of a finite observation of white swans, to the 
theory that all swans are white, not that they believe this to be truth-preserving, or even that they believe this to be a good way of reasoning.

Many things fall under the umbrella of this definition of implication. Bayesian confirmation theory is one case (see for example Talbott, 2016). If some evidence were to raise the probability of a select hypothesis being true, that piece of evidence would be said to confirm that hypothesis. This notion of confirmation would be a type of implication. The evidence, which is itself a theory, implies through very specific rules of implication (Bayes' theorem and conditional probabilities) the hypothesis, which is also a theory. As such, implication, as used here, is a broad term that is not confined to truth-preservation or logical necessity. Even evidence can be said to (inductively) imply a hypothesis. The use of the term implication will hereafter refer to this definition and is not to be conflated with the material implication which is truth-preserving.

Often, when people speak of a reason for believing something, they reference some form of implication, but it can be shown that equating reason with implication would be erroneous. To appreciate this, consider the story of Achilles and the tortoise from Carroll (1995). Among other things, the story provides an illustration of some interesting features of implication and highlights why the notion of reason cannot be equated with that of implication. A person such as Achilles may say they believe in a proposition $B$ because (for the reason that) they believe in a proposition $A$ and they believe that $A \supset B$. Achilles may further say that the reason for his belief of $B$ is his belief of $A$, his belief that $A \supset B$, and modus ponens. So, it seems that Achilles has a logic with modus ponens as a rule of implication. We may then say that Achilles implies $B$ from $A$ and that this implication is a reason for Achilles.

$$
A, A \supset B \rightarrow B
$$

This would be well and fine, but the tortoise has something else in mind. The tortoise, as Carroll illustrates, may accept $A, A \supset B, B$, modus ponens (effectively accepting the above implication), and yet the tortoise may still refrain from saying that the implication is a reason for believing $B$. The tortoise may accept $B$ for entirely different reasons, the implication not being one of them. It's important to note that the tortoise's position does not invalidate the implication; the implication holds no matter what the tortoise thinks about it, because the implication $A, A \supset B \rightarrow B$ is an analytic statement. It is analytic as it is a statement concerning a relation between propositions under a certain logical framework; it says nothing about any contingent state of affairs. However, there remains a question: why does Achilles take the implication as a reason for believing $B$, while the tortoise, who also accepts the implication, does not take it as a reason for believing $B$ ? Achilles may think that the tortoise is a bit slow in his thinking and that he needs to spell out his deduction to help the tortoise understand how modus ponens applies by writing an additional statement in the deduction:

$$
A, A \supset B,(A \wedge(A \supset B)) \supset B \rightarrow B
$$

The tortoise still agrees with this new implication, but merely reiterates that it still does not give him a reason to believe $B$. Achilles may continue clarifying this implication ad infinitum by introducing more clarifications without ever convincing the tortoise. So, what differentiates Achilles and the tortoise, when they hold the same beliefs and agree on everything, except that the implication is a reason for believing $B$ ? One way of viewing the story of Achilles and the tortoise is through the distinction between logic and meta-logic (see for instance Haack, 1976, p. 115). However, I will take a different route by adopting Harman's distinction between implication and inference, and argue that inferences, and not implications, form the basis of reasons. To that end, let us first consider the notion of inference.

\section{Inference}

Making an inference is usually viewed as the mental (as opposed to computational) act of drawing a conclusion from some premises. More abstractly, in order to be distinguished from an implication, an inference can be thought of as a movement of thought, or in the case of epistemology, a movement from certain beliefs to other beliefs. 
These vague characterizations seem to be intuitive in the literature (see for instance Neta, 2013, p. 388; Boghossian 2018, p. 55). For Harman (1986, pp. 3-6), implications alone do not explain revisions of an agent's beliefs; inferences are what an agent makes in order to revise one's beliefs. Following from the previous section, implications are merely analytic descriptive statements, while inferences seem to be subjective to the agent. Although Achilles used implication to revise his beliefs, the tortoise supposedly did not. Inferences and implications are still formally or structurally equivalent: they are both logical and rule-governed, although an agent may have rules of implication that differ from their rules of inference.

Thus, Achilles took his initial beliefs $A$ and $A \supset B$, and then his belief in the implication $A, A \supset B \rightarrow B$ as a justification for believing $B$. He inferred $B$ from the rest. Contrary to Achilles, the tortoise did not infer $B$. There was no "movement of thought" for the tortoise going from the premises of the implication to its conclusion. This shows that, although frequently equated, an agent's rules of implication may differ from their rules of inference. Perhaps it is common to take rules of implication as rules of inference; that seems to be what Achilles did. However, for the tortoise who accepted the implication but denied the inference, his rules of implication do not correspond to his rules of inference.

If this is the case, can we simply forget about implication and focus on inference? Possibly, but I think there is more to say in this story. Although implication seems to refer to something analytic, inference refers to some subjective dispositions that the agent possesses, and some form of justification. Justification is a normative concept, and the rules of inference that an agent follows seem to be based around normative beliefs, i.e. beliefs concerning when one should revise their beliefs. So, it seems that although both implications and inferences share a similar structure, as both follow their rules of implication/inference, they are epistemologically distinct. Implication is descriptive, whereas inference is normative. Just as before, I will separate the descriptive and normative components: implication is taken to be descriptive just like causal theories of the basing relation, while inference is taken as normative just like doxastic theories of reason.

Before continuing this investigation into inferences, I will briefly discuss some fundamental differences between descriptive and normative statements. In scientonomy, all statements are either definitional, descriptive, or normative. Normative statements differ from definition and descriptive statements in two ways. First, normative statements are not about states of affairs or matters of fact, but instead concern modalities towards actions or intentionalities. Secondly, normative statements can carry normative force. Although an agent can take an epistemic stance towards all types of statements, only normative statements can be employed, i.e. followed in practice (Barseghyan, 2018; for a discussion of how normative rules can be applied to rational thinking see Over, 2004, pp. 5-6.) Employment is the scientonomic term for the "normative force" that normative propositions may carry. For example, one can accept that one should not kill, and one can also follow that dictum by having one's actions motivated by this principle, i.e. by employing that norm. The same cannot be said about descriptive statements as they cannot be employed. One can try to say that one employs the descriptive proposition "water freezes at $0^{\circ} \mathrm{C}$ " when predicting the solidity of a skating rink based on a weather forecast, but this would not be a case of employment, rather it would be a case of scientonomic use (Barseghyan, 2015, pp. 30-42).

\section{Reasons}

Our point of departure for explicating a scientonomically acceptable notion of reason is the normative notion of inference. I will examine two views of normative inference before presenting my own notion of normative inference, which will allow me to introduce the notions of reason, sufficient reason, and support.

Considering inferences as normative is not new. Broome (1999) used his normative requirements to explain reasoning. MacFarlane (2004), following Broome, took a more in-depth look at possible ways one can consider inferences normatively. At the bottom line, inferences were thought to be forms of bridge principles - principles that generate normative statements from descriptive statements (for more discussion and examples see MacFarlane, 2004, pp. 5, 7, 22, 24). It is also widely accepted that no bridge principle is analytically true, so bridge principles are synthetic and relative to the agent (for a more in-depth logical look see Schurz, 1997, pp. 
11, 276-285). For MacFarlane, a bridge principle would follow the abstract form "if $A, B$ entails $C$, then (normative claim about believing $A, B$, and $C$ )" (MacFarlane, 2004, p. 6). MacFarlane went on to consider eighteen different bridge principles by varying the normative claims (modalities) and the doxastic claims about $A, B$, and $C$. Broome chose one type of bridge principle that he found particularly effective, namely that "if $p$ implies $q$, one ought to see to it that when $p$ is the case, $q$ is the case", which he called normative requirement, or that " $p$ requires $q$ " (Broome, 1999, p. 406). MacFarlane also considered Broome's normative requirement as the best type of bridge principle of the eighteen he considered (MacFarlane, 2004, p. 13). However, I believe there is a problem in both Broome and MacFarlane's approach that leads them to reject the bridge principles that do not coincide with Broome's normative requirement. Furthermore, I do not find that normative requirement is particularly enlightening about how agents reason.

The problem is that in the antecedent of their bridge principles, they take an alethic statement about implication, rather than a doxastic one, i.e. $A$ and $B$ entail $C$, but not that the agent believes that $A$ and $B$ entail $C$. Their assumption of logic is also unclear. Both Broome and MacFarlane take the truth of the entailment to rule out "irrational" bridge principles that don't respect the truth of the antecedent. However, if the antecedent is instead replaced with the belief of an implication, which may be fallible, there is no longer a reason to reject certain bridge principles. Also, the bridge principle of a normative requirement, which states that if $q$ follows from $p$ then one ought to contingently infer $q$ from $p$, does not add much to the understanding of an agent's inference. It is simply a statement that truth-preserving rules of implication are also rules of inference, which is not necessarily the case, as was seen in the example of Achilles and the tortoise.

Now, the bridge principle, or type of inference, I wish to present is one based on an agent's accepted theories, accepted implications, and employed norms. A reason will be said to be a stricter condition on this normative inference. The notion of sufficient reason can be defined as follows:

\begin{tabular}{|l|}
\hline Sufficient Reason $\equiv$ \\
\hline An agent takes theory $A$ to be a sufficient reason for \\
(accepting) theory $B$ iff the following four conditions are met: \\
(1) The agent accepts $A$. \\
(2) The agent accepts that $A \rightarrow B$. \\
(3) The agent employs $\varepsilon$. \\
(4) The agent accepts $(\varepsilon, A, A \rightarrow B) \rightarrow_{\varepsilon}$ (Should accept $\left.B\right)$.
\end{tabular}

Let's unpack this. Condition (1) is self-explanatory: the agent takes $A$ to be the best available answer to a certain question (Barseghyan, 2018, p. 31). Condition (2) refers to the earlier discussion of implication: the descriptive theory that " $B$ follows from $A$ " is accepted by the agent under their rules of implication. Furthermore, while $A$ and $B$ are not necessarily descriptive or normative, the statement $A \rightarrow B$ is necessarily descriptive as it describes an implication and does not prescribe any action. Importantly, it is descriptive even if the implication is from normative premises to a normative conclusion. This condition is what is typically referred to in causal theories of reasons as the awareness of logical relations. Condition (3) says that the method is employed by the agent, i.e. it constitutes the actual expectations of the agent. Condition (4) is more interesting. The statement "should accept $B$ " is a claim that prescribes the action of acceptance. It postulates that the agent accepts that the statement "should accept $B$ " follows from the method, $A$, and $A$ implies $B$. In other words, the agent accepts that the propositional contents of (1) and (2) alongside the employed norms imply a normative claim that is equivalent to scientonomic acceptance (Barseghyan, 2018, p. 31). It's very important to note here that this is not any form of normative naturalism, as the employed norms in the premises of the implication are necessarily normative. Also note that the implication in (4) is with respect to the rules of norm implication specific to the agent's methods. This implication involves both descriptive and normative premises in order to imply its normative conclusion. Additionally, the implication may follow rules of implication that are different from typical descriptive implications for the agent. One will usually need to apply some bridge principle, which is itself a rule of 
implication, in order to imply a normative conclusion, whereas this is not the case for implications between descriptive theories. This is to be contrasted with the implication in (2) which may be purely between descriptive theories, and not have any bridge principle as a rule.

Now, if only conditions (1) and (2) are satisfied, one can say that $A$ supports $B$, or, loosely, $A$ is an insufficient reason for (accepting) $B$. If only conditions (2), (3), and (4) are satisfied, then $A$ is a reason for $B$. Lastly, if only conditions (1), (2), and (4) are satisfied, then $A$ is said to normatively infer $B$. Let's consider each of these notions in turn.

First, the satisfaction of conditions (1) and (2) means that $A$ supports $B$. The definition is as follows:

\begin{tabular}{|c|}
\hline Support $\equiv$ \\
\hline $\begin{array}{c}\text { An agent takes theory } A \text { to be supporting theory } B \\
\text { iff the agent accepts } A \text { and accepts that } A \rightarrow B .\end{array}$ \\
\hline
\end{tabular}

The term support aims to capture a theory "giving evidence" to believe in another theory. For example, a confirming observation (in the Bayesian sense) may imply a hypothesis, and hence be a reason for accepting that hypothesis. This notion of support is stronger than that of implication as it also requires the "premises" of the implication to be accepted. However, support may be insufficient to accept the hypothesis on its own, as it may not satisfy the agent's employed norms.

In contrast, the satisfaction of conditions (2), (3), and (4) means that $A$ is a reason for $B$. Here is the definition:

\begin{tabular}{|c|}
\hline Reason $\equiv$ \\
\hline $\begin{array}{c}\text { An agent takes theory } A \text { to be a reason for theory } B \\
\text { iff the agent accepts that } A \rightarrow B, \text { employs } \varepsilon, \text { and } \\
\text { accepts }(\varepsilon, A, A \rightarrow B) \rightarrow_{\varepsilon}(\text { Should accept } B) .\end{array}$ \\
\hline
\end{tabular}

It follows from this definition that sufficient reason is a subtype of reason. This is exemplified by the term reason aiming to capture the contingent (but not necessarily actual) conditions required for accepting a theory. For example, if some of the novel predictions of string theory were confirmed, this would be a reason for the physics community to accept string theory. However, the experimental confirmation of these novel predictions requires currently unavailable technology. Since these predictions have not been confirmed, (1) remains unsatisfied, which means that the physics community doesn't have a sufficient reason to accept string theory. Yet, we can still speak hypothetically and say that the physics community would have a sufficient reason for accepting string theory had those confirmations been observed and accepted. Thus, those potential confirmations would be a reason for accepting string theory, but they are not a sufficient reason for the current physics community.

To illustrate the difference between support and reason, let's return to the case of Achilles and the tortoise. This case shows a clear difference between agents who accept the same descriptive theories but have different (employed) norms. Both Achilles and the tortoise accept the premises and the implication, i.e. conditions (1) and (2) are satisfied for both of them. Yet, while Achilles infers the conclusion of the implication, the tortoise does not. This can be attributed to the fact that Achilles has his employed method satisfied, while the tortoise does not have his method satisfied. This means that condition (3) is satisfied for Achilles but not for the tortoise. So, Achilles has a sufficient reason for accepting the conclusion, while the tortoise only has a case of support. This would then imply a difference in employed norms between the tortoise and Achilles, since they both use the same rules of implication.

Finally, the notions of sufficient reason, reason, and support are not to be conflated with the notion of normative inference. If only conditions (1), (2), and (4) are satisfied, then $A$ is said to normatively infer B. Thus, the notion of normative inference can be defined as follows: 


\begin{tabular}{|c|}
\hline Normative Inference $\equiv$ \\
\hline An agent takes theory $A$ to normatively infer theory \\
$B$ iff the agent accepts $A$, accepts that $A \rightarrow B$, and \\
accepts $(\varepsilon, A, A \rightarrow B) \rightarrow_{\varepsilon}$ (Should accept $B$ ). \\
\hline
\end{tabular}

The major difference between the notions of sufficient reason and normative inference is that while normative inference involves arbitrary norms, reason involves employed methods. This clearly distinguishes reasons as a form of drivers of acceptance rather than mere normative rules for theory acceptance. The notion of normative inference may be used to identify norms that an agent does not employ, or to discuss an agent's hypothetical stance on a certain theory had they employed a different method. For example, a historian may ask what the Aristotelian-Medieval community would have done in the face of Galileo's observations had they been employing a hypothetico-deductive method.

This notion of normative inference is similar to a common form of bridge principle that is criticized by a number of authors. Harman, Broome, and MacFarlane all argue against a certain type of bridge principle that is apparently intuitive, and very much resembles our notion of normative inference. It was named by MacFarlane as the $c_{o+}$ (ought to positively believe in the consequent) bridge principle and is formulated as "if $A$ and $B$ entail $C$, then if you believe $A$ and you believe $B$, then you ought to believe $C$ " (MacFarlane, 2004, p. 7). While the form is very similar to my definition of normative inference, there are subtle differences that help us avoid two major criticisms.

One major criticism of that bridge principle is that if it holds, then if one believes in $A$, then one ought to believe in $A$, since $A$ entails $A$. This is problematic, as frequently one believes things that one ought not necessarily

Although a case could be made that everything one believes in is something one ought to believe in, but arguments for this position will be omitted.

norms do not imply that they ought to accept $A$ even if they believe $A$. Notice however that the agent's norms might just as well be the $c_{o+}$ bridge principle which would run into this criticism, but that would be a contingent state of the agent's employed norms, and not a necessary one. Hence, this criticism seems to be avoided.

Another major criticism is that if one reaches an absurd conclusion from one's beliefs, then instead of accepting the conclusion, one should reject, or at least deny the consequent of the $c_{o+}$ bridge principle. Since the $c_{o+}$ bridge principle includes logical entailment as one of its premises rather than the acceptance of an implication, we do not need to be committed to believing the consequent whenever we find an implication. Sometimes our norms would tell us otherwise, even if the implication at hand is a truth-preserving one. This may be evident in the case of the inconsistency between quantum mechanics and general relativity. Although the two theories entail a contradiction and, by the principle of explosion, entail any proposition, we still accept those two theories. Even then, we do not accept all propositions entailed by the conjunction of quantum mechanics and general relativity; such an acceptance would go against our method (norms). Perhaps our norms suggest that we ought not to accept everything implied by the inconsistent conjunction of these two theories, but this is mere speculation concerning the norms in contemporary physics. As such, this criticism does not hold water.

With this new taxonomy at hand we can now deduce what can be called the sufficient reason theorem. Since the notion of sufficient reason assumes that the respective method is employed (i.e. condition (3) is satisfied), it can be shown that, by the second law, the agent accepts a theory when there is a sufficient reason for accepting it. The proof is as follows. If an agent has a sufficient reason for accepting a theory, then (4) is satisfied. Condition (4) implies that what is being reasoned for satisfies the employed method, i.e. the actual expectations of the agent. This is because (4) says that "should accept B" follows from the employed method and other accepted theories. By the second law, if the employed method is satisfied, then the theory will be accepted: 
Sufficient Reason $\equiv$

An agent takes theory $A$ to be a sufficient reason for (accepting) theory $B$ iff the following four conditions are met:

(1) The agent accepts $A$.

(2) The agent accepts that $A \rightarrow B$.

(3) The agent employs $\varepsilon$.

(4) The agent accepts $(\varepsilon, A, A \rightarrow B) \rightarrow_{\varepsilon}$ (Should accept $\left.B\right)$. $2^{\text {nd }}$ Law: Theory Acceptance

If a theory satisfies the acceptance criteria of the method employed at the

time, it becomes accepted into the mosaic; if it does not, it remains unaccepted; if assessment is inconclusive, the theory can be accepted or not accepted.

This notion of reason also offers some insight into the third law. According to the current formulation of the third law, a method becomes employed only when it is deducible from some subset of other employed methods and accepted theories of the time (Sebastien, 2016, p. 4). The term "deducible" appears quite vague in the current formulation of the law, but to be "deducible from some subset of other employed methods and accepted theories of the time" sounds very much like a type of implication. If we consider $\alpha$ to be the set of all accepted theories of an agent, then we can cast the deducibility statement of the third law as follows:

$$
\alpha, \varepsilon \rightarrow m
$$

Here, the implication is assumed to be under the agent's notion of implication, rather than some universal ahistorical notion of implication. The accepted theories of the agent and the employed norms of the agent imply, by the agent's notions of implication, some arbitrary method $m$ which is itself a normative theory. Thus, the third law can be stated as: a method $m$ becomes employed only when $\alpha, \varepsilon \rightarrow m$. This makes the third law much clearer. Furthermore, it points out that the agent need not take an epistemic stance towards that implication (previously "deducibility"). The third law seems to be purely causal and not doxastic; the agent does not justify their new employment according to the third law, as it is merely a descriptive implication that is satisfied whenever a new method becomes employed. Since the third law does not explicitly contain a notion of acceptance by the agent in its current formulation, or the formulation suggested here, it seems to bear little connection to reasons, which may be either a drawback or a virtue of its formulation. I will leave further discussion of the third law in the context of reasons to future research.

\section{Examples and Illustrations}

Having presented a scientonomic definition of reason, I will now illustrate it with some examples. It should be noted that these examples are meant purely for illustration purposes and do not involve any substantive historical claims. However, I do hope that they demonstrate some of the non-trivial uses of reasons.

The first example is the already mentioned case of the Busemann-Petty problem. The epistemic agent Zhang took his 1994 mathematical proof as a reason for accepting a negative answer to the Busemann-Petty Problem in four dimensions. It was a sufficient reason since Zhang accepted the premises in his proof satisfying (1). Zhang accepted that the mathematical deduction leading to a negative answer was valid and hence supported the negative answer satisfying (2). Zhang's employed method of accepting the results of mathematical deductions meant that Zhang should believe in a negative answer to the Busemann-Petty Problem which satisfied both (3) and (4). 
Hence, Zhang had a sufficient reason for believing in a negative answer to the Busemann-Petty problem. So, by virtue of the sufficient reason theorem, Zhang accepted a negative answer to the Busemann-Petty Problem.

In 1998, Koldobsky provided an alternative proof that led to the opposite answer. Zhang also discovered a mistake in his deduction in 1999. This led Zhang to reject his mathematical proof, meaning that (2) was no longer satisfied. This meant that his mathematical proof was no longer a reason for a negative answer. Nowadays, Koldobsky's proof is a reason for accepting the positive answer as it satisfies (1)-(4), even to Zhang. One thing to note is that with the current framework of the laws of scientific change, one will have to invoke other arguments in explaining why Zhang rejected a negative answer, as we are unable to say that he no longer accepted it because he no longer had a reason to (which is what one might intuitively want to do). Instead, one would need to invoke the theory rejection theorem which I have not been able to deduce from the sufficient reason theorem.

Our notion of reason can be further illustrated by applying it to the case of the Higgs boson. The question of how force carriers get their mass without breaking symmetries was prominent in the early 1960s after observed spontaneous symmetry breakings (electroweak symmetry breaking) in Ginzburg-Landau and Bardeen-CooperSchriefer superconductivity theories (Wells, 2017). In 1964, Higgs answered this question by postulating a quantum field (the Higgs field) that explained the spontaneous symmetry breaking in superconductivity (the Higgs mechanism) and also explained why certain bosons have mass. It was, however, not clear at this point whether it was necessary for the Higgs boson to exist. Higgs proposed the hypothesis that the Higgs field is elementary (i.e. not made of more fundamental constituents), a theory which many physicists regarded with skepticism. Thus, Susskind, Veltman, and Georgi opposed the existence of the Higgs boson. Susskind even considered it a flaw of the Weinberg-Salam theory (the Standard Model). In 2000, competing and incompatible theories such as top quark condensation were proposed by skeptics of the Higgs boson. There were many opponents to the existence of a Higgs boson stressing that in addition to it not having been discovered, it also led to the hierarchy problem. Yet others were all the while embedding the Higgs field in more exotic theories (supersymmetry, string theory) to make it viable. There was also the problem of the Higgs boson's mass itself and the difficulty in identifying it which would require actually discovering the boson. By 2011 however, it was at least clear that Higgs-less theories failed to be accurate descriptions of nature. Finally, the Higgs discovery announcement, after the herculean project at the LHC, was announced in July 2012, marking its entry into university textbooks (Schwartz, 2013, pp. 575579).

With our new definition of reason at hand, we can interpret this historical episode as follows. From 1964, the theory of Higgs boson became pursued as an answer to the question of electroweak symmetry breaking. It was initially met with resistance, as it was not clear whether or not it was a necessary feature of the Standard Model. However, by 1979 it was accepted that its existence followed from the Standard Model. In other words, the standard model, which was accepted and thus satisfying (1), was shown to (deductively) imply the Higgs boson, satisfying (2). However, conditions (3) and (4) were not yet met as the employed method required confirmation of novel predictions, not deductions from the Standard Model. After its discovery at the LHC in 2012, it was finally accepted by the physics community, indicated by its introduction into university textbooks as early as 2013 (Schwartz, 2013, pp. 575-579). The experimental confirmation of the Higgs boson satisfied the requirements of the employed method of the time, thereby satisfying condition (4), which meant that there was now a sufficient reason to accept the existence of the Higgs boson. By the sufficient reason theorem, the Higgs boson was accepted.

I believe that the Higgs boson case is a typical example of how a theory becomes accepted as a reason for another theory. Initially, there are supporting theories for a hypothesis that are still insufficient for accepting that hypothesis, i.e. (1) and (2) are satisfied, but not (4). It is only after, when the support is bolstered by newly accepted theories (i.e. by LHC observations and experiments), that the employed method is satisfied, and the community has sufficient reason to accept the new theory. This kind of strengthening of support via newly acquired theories (observations, theoretical results, etc.) I anticipate will be the most common scenario leading to something being accepted with a sufficient reason, when it only had support before.

After having considered those two historical examples, I now move to addressing two theoretical questions, the first of which concerns the reason for accepting a definition. What could possibly be the reason for accepting 
a definition? It may seem that there is no justification for a definition, as an agent can arbitrarily choose what to call certain terms. However, definitions usually have a context of descriptive and normative theories in which they are used or presupposed. Typically, when a theory is accepted, it comes bundled with its own definitions. The definitions are then accepted for the same reason the set of theories they are bundled in is accepted. In this sense, definitions are accepted by consequence of their context, and not as standalone theories. In more detail, suppose an agent has a reason for accepting theory $P$ and does so. Theory $P$ includes a definition as part of its set of propositions. What is the reason for accepting that definition? It would be the same reason as for accepting the whole theory, since they come as part of a package. So, definitions, at least in what seems to be the common case, are not independently reasoned for from the theory they come in.

For example, consider the acceptance of Newtonian mechanics. Along with many descriptive theories, Newtonian mechanics included definitions, one of which was the definition of the term "force". This definition of "force" was not accepted in isolation. It was only its place within the wider Newtonian theory that made it acceptable. If one had a reason to accept Newtonian mechanics, then one also had a reason to accept all the definitions formulated in Newtonian mechanics, including that of force.

The second theoretical question concerns reasons for theories that are considered self-evident. Suppose an agent accepts a certain theory as foundational, self-evident, basic, or not requiring justification. What, if anything, is the agent's reason for accepting this theory? An agent could say that they accept that theory because it is selfevident (i.e. its self-evidence is the reason). But then a question arises as to what the reason for it being selfevident is, and then what the reason for that reason is, and so on. Eventually, one would necessarily arrive at a theory that has no reason, engage in infinite regress, or fall into circular reasoning. The case of infinite regress, while theoretically conceivable, is practically unattainable for epistemic agents with finite capacity. The two other scenarios, however, seem to be practically attainable. Thus, in the case of circularity, an agent can consider theory $A$ as a reason for theory $B$ while also considering theory $B$ as a reason for theory $A$. Under circular reasoning, it is not the case that each theory could have been accepted in virtue of the other. Indeed, under the supposition that both $A$ and $B$ came to be accepted with a sufficient reason, by the sufficient reason theorem, theory $A$ could not have come to be accepted without theory $B$ already being accepted, while theory $B$ could not have come to be accepted without theory A already being accepted. This is because condition (1) requires them to have already been accepted in order to serve as sufficient reasons. Similarly, in the case where a theory is accepted for no reason, it certainly was not accepted through the sufficient reason theorem. Importantly, these scenarios do not violate the sufficient reason theorem, as the theorem doesn't say that having a sufficient reason is the only way for a theory to become accepted.

This raises an important question: what is the mechanism of theory acceptance in the cases when a theory becomes accepted without a sufficient reason, i.e. in the cases of circularity or theories without a reason? I will not attempt to address this question in this paper but will suggest it as legitimate topic of scientonomic inquiry (provided that the sufficient reason theorem is accepted).

\section{Conclusion}

I have introduced the notion of reason into the scientonomic context under the abstractionist thesis as a type of theory towards which an epistemic stance is taken. According to the suggested definition, theory $A$ is said to be a reason for theory $B$ if and only if the agent accepts that $A \rightarrow B$, the agent employs some method $\varepsilon$, and accepts that $(\varepsilon, A, A \rightarrow B) \rightarrow_{\varepsilon}$ (Should accept $B$ ). In addition, I have provided scientonomic definitions for the notions of sufficient reason, support, and normative inference. It is clear that this new taxonomy can have major theoretical implications. One such implication is the sufficient reason theorem, which is deduced from the second law and the definition of sufficient reason. Another implication is the clarification of the notion of deduction in the third law. Other theoretical and observational implications of the new taxonomy are yet to be explored. At this stage, my hope is that there is a good reason for accepting this taxonomy into the ontology of scientific change. 


\section{Suggested Modifications}

\section{[Sciento-2019-0009]}

Accept the following definition of implication:

- Implication $\equiv$ a logical transition from one theory to another.

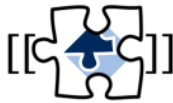

\begin{tabular}{|c|}
\hline Implication $\equiv$ \\
\hline $\begin{array}{c}\text { A logical transition from } \\
\text { one theory to another. }\end{array}$ \\
\hline
\end{tabular}

\section{[Sciento-2019-0010]}

Accept the following definitions of sufficient reason, reason, support, and normative inference:

- Sufficient Reason $\equiv$ an agent takes theory $A$ to be a sufficient reason for (accepting) theory $B$ iff the following four conditions are met:

(1) The agent accepts $A$.

(2) The agent accepts that $A \rightarrow B$.

(3) The agent employs $\varepsilon$.

(4) The agent accepts $(\varepsilon, A, A \rightarrow B) \rightarrow_{\varepsilon}$ (Should accept $\left.B\right)$.

- Support $\equiv$ an agent takes theory $A$ to be supporting theory $B$ iff the agent accepts $A$ and accepts that $A \rightarrow B$.

- Reason $\equiv$ an agent takes theory $A$ to be a reason for theory $B$ iff the agent accepts that $A \rightarrow B$, employs $\varepsilon$, and accepts $(\varepsilon, A, A \rightarrow B) \rightarrow_{\varepsilon}$ (Should accept $B$ ).

- Normative Inference $\equiv$ An agent takes theory $A$ to normatively infer theory $B$ iff the agent accepts $A$, accepts that $A \rightarrow B$, and accepts $(\varepsilon, A, A \rightarrow B) \rightarrow_{\varepsilon}$ (Should accept $B$ ).

\begin{tabular}{l} 
Sufficient Reason $\equiv$ \\
\hline An agent takes theory $A$ to be a sufficient reason for (accepting) \\
theory $B$ iff the following four conditions are met: \\
(1) The agent accepts $A$. \\
(2) The agent accepts that $A \rightarrow B$. \\
(3) The agent employs $\varepsilon$. \\
(4) The agent accepts $(\varepsilon, A, A \rightarrow B) \rightarrow_{\varepsilon}$ (Should accept $\left.B\right)$. \\
\hline
\end{tabular}

\begin{tabular}{|c|}
\hline Support $\equiv$ \\
\hline $\begin{array}{c}\text { An agent takes theory } A \text { to be supporting theory } B \\
\text { iff the agent accepts } A \text { and accepts that } A \rightarrow B .\end{array}$ \\
\hline
\end{tabular}

\begin{tabular}{|c|}
\hline Reason $\equiv$ \\
\hline An agent takes theory $A$ to be a reason for theory $B$ \\
iff the agent accepts that $A \rightarrow B$, employs $\varepsilon$, and \\
accepts $(\varepsilon, A, A \rightarrow B) \rightarrow_{\varepsilon}$ (Should accept $\left.B\right)$. \\
\hline
\end{tabular}

\section{Normative Inference $\equiv$}

An agent takes theory $A$ to normatively infer theory

$B$ iff the agent accepts $A$, accepts that $A \rightarrow B$, and accepts $(\varepsilon, A, A \rightarrow B) \rightarrow_{\varepsilon}$ (Should accept $\left.B\right)$. 


\section{[Sciento-2019-0011]}

Provided that modification [Sciento-2019-0010] is accepted, accept the sufficient reason theorem and its deduction from the definition of sufficient reason and the second law:

- Sufficient Reason theorem: a theory becomes accepted by an agent, when an agent has a sufficient reason for accepting it.

\section{Sufficient Reason $\equiv$}

An agent takes theory $A$ to be a sufficient reason for (accepting) theory $B$ iff the following four conditions are met:

(1) The agent accepts $A$.

(2) The agent accepts that $A \rightarrow B$.

(3) The agent employs $\varepsilon$.

(4) The agent accepts $(\varepsilon, A, A \rightarrow B) \rightarrow_{\varepsilon}$ (Should accept $B$ ).

\section{$2^{\text {nd }}$ Law: Theory Acceptance}

If a theory satisfies the acceptance criteria of the method employed at the time, it becomes accepted into the mosaic; if it does not, it remains unaccepted; if assessment is inconclusive, the theory can be accepted or not accepted.

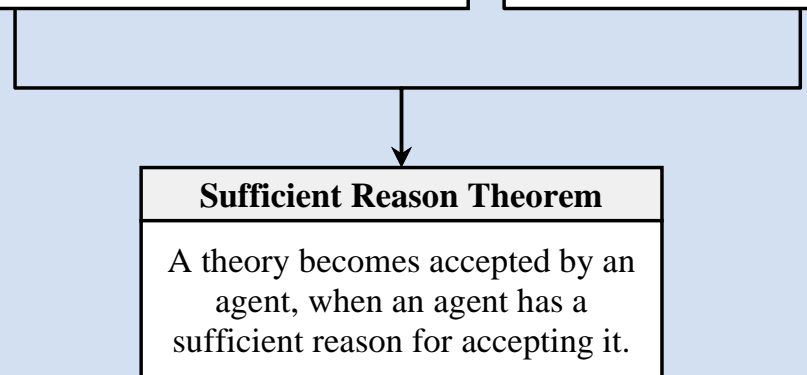

Accept the following question as a legitimate topic of scientonomic inquiry:

- Theory Acceptance without Sufficient Reason: how do theories become accepted without a sufficient reason, i.e. in the cases of circularity or theories without a reason?

\section{References}

Barseghyan, H. (2015). The Laws of Scientific Change. Springer.

Barseghyan, H. (2018). Redrafting the Ontology of Scientific Change. Scientonomy 2, pp. 13-38. Retrieved from https://scientojournal.com/index.php/scientonomy/article/view/31032.

Bonjour, L. \& Sosa, E. (2003). Epistemic Justification. Blackwell Publishing.

Boghossian, P. (2018). Delimiting the Boundaries of Inference. Philosophical Issues 28, Philosophy of Logic and Inferential Reasoning, pp. 55-69.

Broome, J. (1999). Normative Requirements. Ratio 7(4), pp. 398-419.

Carroll, L. (1995). What the Tortoise said to Achilles. Mind 104(416), pp. 691-693.

Carter, J. A. \& Bondy, P. (Eds.) (2019). Well-Founded Belief: New Essays on the Epistemic Basing Relation. Routledge.

Csáki, C.; Grojean, C.; Pilo, L.; \& Terning, J. (2004). Towards a Realistic Model of Higgsless Electroweak Symmetry

Breaking. Physical Review Letters 92(10), 101802.

Cunningham, J. J. (2019). Is Believing for a Normative Reason a Composite Condition? Synthese 196(9), pp. 3889-3910.

Gregory, A. (2016). Normative Reasons as Good Bases. Philosophical Studies 173, pp. 2291-2310.

Haack, S. (1976). The Justification of Deduction. Mind 85(337), pp. 112-119.

Harman, G. (1986). Change in View: Principles of Reasoning. MIT Press.

Huber, F. (2017). On the Justification of Deduction and Induction. European Journal for Philosophy of Science 7, pp. 507534.

Koehler, D. J. \& Harvey, N. (Eds.). (2004). The Blackwell Handbook of Judgment and Decision Making. Blackwell

Publishing.

Koldobsky, A. (1998). Intersection Bodies in $\mathrm{R}^{4}$. Advances in Mathematics 136(1), pp. 1-14.

Korcz, K. (1997). Recent Work on the Basing Relation. American Philosophical Quarterly 34(2), pp. 171-191.

Korcz, K. (2000). The Causal-Doxastic Theory of the Basing Relation. Canadian Journal of Philosophy 30 (4), pp. 525-550. 
Lord, E., \& Sylvan, K. (2019). Prime Time (for the Basing Relation). In Carter \& Bondy (Eds.) (2019), pp. 141-174.

MacFarlane, J. (2004). In What Sense (If Any) Is Logic Normative for Thought? Manuscript of original presentation at Central Division APA 2004. Retrieved from https://johnmacfarlane.net/normativity_of_logic.pdf.

Neta, R. (2013). What Is an Inference? Philosophical Issues 23, Epistemic Agency, pp. 388-407.

Over, D. (2004). Rationality and the Normative/Descriptive Distinction. In Koehler \& Harvey (Eds.) (2004), pp. 3-18.

Sebastien, Z. (2016). The Status of Normative Propositions in the Theory of Scientific Change. Scientonomy 1, pp. 1-9. Retrieved from https://www.scientojournal.com/index.php/scientonomy/article/view/26947.

Schurz, G. (1997). The Is-Ought Problem. Springer.

Schwartz, M. (2013). Quantum Field Theory and the Standard Model. Cambridge University Press.

Starr, W. (2019). Counterfactuals. In Zalta, E. N. (Ed.) (2019). The Stanford Encyclopedia of Philosophy (Fall 2019

Edition). Retrieved from https://plato.stanford.edu/archives/fall2019/entries/counterfactuals/.

Steinberger, F. (2017). The Normative Status of Logic. In Zalta, E. N. (Ed.) (2017). The Stanford Encyclopedia of

Philosophy (Spring 2017 Edition). Retrieved from https://plato.stanford.edu/archives/spr2017/entries/logic-normative/.

Sylvan, K. (2016a). Epistemic Reasons I: Normativity. Philosophy Compass 11(7), pp. 364-376.

Sylvan, K. (2016b). Epistemic Reasons II: Basing. Philosophy Compass 11(7), pp. 377-389.

Talbott, W. (2016). Bayesian Epistemology. In Zalta, E. N. (Ed.) (2016). The Stanford Encyclopedia of Philosophy (Winter 2016 Edition). Retrieved from https://plato.stanford.edu/archives/win2016/entries/epistemology-bayesian/.

Turri, J. (2009). The Ontology of Epistemic Reasons. Noûs 43(3), pp. 490-512.

Wells, J. D. (2017). Beyond the Hypothesis: Theory's Role in the Genesis, Opposition, and Pursuit of the Higgs Boson. Studies in the History and Philosophy of Modern Physics 62, pp. 36-44.

Zhang, G. (1994). Intersection Bodies and the Busemann-Petty Problem in $\mathrm{R}^{4}$. Annals of Mathematics, Second Series 140(2), pp. 331-346.

Zhang, G. (1999). A Positive Solution to the Busemann-Petty Problem in $\mathrm{R}^{4}$. Annals of Mathematics, Second Series 149(2), pp. 535-543. 